[2] J. B., Moore and B. D. O. Anderson, "A generalization of the Popov crite(3] rion," J. Franklin Inst., vol. 285, pp. 488-492, June 1968 .

3] M. Pai, "Transient stability studies in power systems using LyapunovPopov approach," presented at the 5th Int. IF AC Congr., Paris, June 12-
17, 1972, Paper 265.

\section{Generalized Factorizability Conditions for Stability Multipliers}

\section{K. SUNDARESHAN AND M. A. L. THATHACHAR}

Abstract-Generalized conditions are given for the factorization of a linear convolution operator in a Banach algebra into a cascade of two operators, one causal and the other anticausal, which has application in the input-output stability analysis of feedback systems. These conditions are an improvement over the earlier ones presented by the authors.

\section{Ixtrodictios}

Following the work of Zames and Falb [1] in introducing linear noncausal convolution operators in $L_{2}$ as "multipliers" for the analysis of the $L_{2}$-stability of feedback systems, the problem of determining conditions that ensure a factorization of these multipliers into a cascade of a causal operator and an anticausal operator in $L_{2}$ has gained much prominence. For a restricted class of multipliers, such conditions have been given by Zames and Falb [1] and Willems and Brockett [2]. Improved conditions for a very general class of noncausal operators have recently been reported by the authors [3], by relating the factorizability to certain positivity properties of the operators. The purpose of this correspondence is to exploit certain properties of the multipliers via spectral theory considerations, to obtain less stringent conditions for the factorization.

\section{The Factorization of Operators}

While the notations used in [3] will be followed, a few of the important ones are briefly recapitulated. Let $R, R^{+}$, and $J^{+}$denote, respectively, the real numbers, the nonnegative real numbers, and the nonnegative integers. An operator $H$ in $L_{2}$ is a single-valued mapping of $L_{2}$ into itself. $H$ is said to be positive (strongly positive) if the inequality $\langle x(\cdot), H x(\cdot)\rangle \geq \delta\langle x(\cdot), x(\cdot)\rangle \forall x(\cdot) \in L_{2}$, holds with $\delta=0(\delta>0)$. For linear time-invariant convolution operators $H$ in $L_{2}$ (i.e., $H x(t)=\int_{-\infty}^{+\infty} h(t-\tau) x(\tau) d \tau \forall x(\cdot) \in L_{2}$ ), $\operatorname{Re} H(j \omega)$ $\geq \delta \forall \omega \in R$ with $\delta=0(\delta>0) \Rightarrow$ positivity (strong positivity) of $\vec{H}$. Let $B$ denote the commutative Banach algebra of linear timeinvariant bounded convolution operators in $L_{2}$, with an identity $E$. $B_{c} \subset B \ni H \in B_{c} \Rightarrow H$ is causal and $B_{a c} \subset B \ni H \in B_{a c} \Rightarrow H$ is anticausal. It is easy to observe that $B=B_{c} \cup B_{a c}$. An operator $H \in B$ is said to be regular in $B$ if $H^{-1} \in \mathbb{B}$. Let $\alpha(B, B)$ denote the space of all continuous linear maps of $B$ into itself and let $E_{\alpha}$ be the identity of $\alpha(B, B)$. Let $P_{-}$be a projection on $B$ and let $P_{-}=E_{\alpha}-$ $P_{+}$.

The problem is to determine conditions that are sufficient to ensure that, for an operator $H \in B$, there exist elements $M_{-}$and $M_{+}$of $B$ such that: 1) $M_{-} \in \mathbb{B}_{a c}$ and $M_{+} \in B_{c}$;2) $M=M_{-} M_{+}$; and 3) $M_{+}$ and $M_{-}$are regular in $B_{c}$ and $B_{a c}$, respectively. We will call this a "compatible factorization" of $M$.

The authors' earlier result [3] contained a condition of the form $M \in \mathbb{B}$ is strongly positive $\Rightarrow M$ admits a compatible factorization with $M_{+}=\exp \left[P_{+} \log M\right]$ and $M_{-}=\exp \left[P_{-} \log M\right]$. A proof of this involved the establishment of $\log i f \in \mathbb{B}$ via the DunfordTaylor integral (DTI)

$$
\log M=\frac{1}{2 \pi j} \int_{\Gamma}(\log \xi) \quad Q(\xi ; M) d \xi
$$
stitute of Science, Bangalore 12, India. where $R(\xi ; M)=(\xi E-M)^{-1}$ is the resolvent of $M, \Gamma$ is a simple closed curve in the complex plane enclosing the spectrum of $M$, and $j=\sqrt{-1}$.

While this result is a fairly simple one, the requirement of strong positivity prohibits many useful operators from being employed as multipliers. ${ }^{1}$ Hence an improved result that overcomes this requirement would be very useful and such a result is presented in the following lemma.

Lemma: If $M \in Q$ is positive and is regular in $B$, then $M$ admits a compatible factorization with $M_{+}=\exp \left[P_{+} \log M\right]$ and $M_{-}=$ $\exp \left[P_{-} \log M\right]$.

Proof: The proof follows along the same lines as in [3]. The crucial step is the choice of $r$, the contour of integration in DTI, inside a simply connected domain $D$ in the complex plane such that $D \supset \sigma(M)$, the spectrum of $M$, but avoids the negative real line including the origin (for details, see [3]). Now $M$ positive $\Rightarrow \sigma(M)$ is contained in the closed right-half plane. Also $M$ is regular in $B \Rightarrow$ the origin $O_{c} \notin \sigma(M)$. The latter assertion is a result of the following arguments. $M$ is one-to-one $=>O_{c} \notin \sigma_{p}(M)$, the point spectrum; $M$ is onto $\Rightarrow O_{c} \notin \sigma_{r}(M)$, the residual spectrum; and $M^{-1}$ is bounded $\Rightarrow$ $O_{c} \notin \sigma_{\epsilon}(M)$, the continuous spectrum (see Dunford and Schwartz $\left[4\right.$, p. 580]). Further, $\sigma(M)$ is a closed set $\Rightarrow O_{c}$ cannot be the limit of a sequence of spectrum points (for example, of the form $\{1 / n\}, n \in$ $J^{+}$). Hence a choice of $\Gamma$, as required, is possible.

The rest of the proof involves simple arguments to show that $\log M$ (defined by DTI) $\in \mathbb{B}$, which will be omitted due to its being available in [3].

\section{Disctission of the Result}

The usefulness of the lemma imposing the conditions of positivity and invertibility on the operators $M$, which are intended to be used as multipliers, may not be apparent unless one goes into the detailed properties of such operators. Note that these are precisely the two necessary conditions to be satisfied by an operator $M \in \mathbb{B}$ to be qualified as a "stability multiplier;" for an $M \in \mathbb{B}, M^{-1} \in \mathbb{B}$ is necessary for the system transformation by the introduction of $M$, without violating the nature of the input-output relation of the system [5], while $\operatorname{Re} M(j \omega) \geq 0 \forall \omega \in R$ is the fundamental requirement in the multiplier theory [6]. Thus the importance of the present lemma lies in its assertion- "every stability multiplier is factorizable."

It is felt that the generalized condition for the factorizability of multipliers provides an easy derivation by functional analysis techniques, for some of the basic results in stability theory. One such result is the sufficiency part of the multiplier version of the Nyquist criterion for systems containing an operator $G \in Q_{\mathrm{c}}$ around which a negative feedback loop with constant gain in $[0, \infty)$, is built. Note the ease with which the following result can be established, using the positivity theorem [1]. The system under consideration is $L_{2}$-stable if there exists an operator $M \in B$ satisfying the twin conditions: 1) $\operatorname{Re} M(j \omega) \geq 0 \forall \omega \in R$; and 2) $\operatorname{Re} M(j \omega) G(j \omega) \geq \delta>0 \forall \omega \in R$. It has been shown in many earlier publications [6], [7] that similar frequency-domain inequalities are also necessary for the system stability.

The factorization problem, besides being of crucial importance in stability theory, has been playing a fundamental role in various other branches of system theory such as stochastic filtering theory, optimal control, etc. [5], [8]. The main reason for obtaining the present result in terms of the positivity and the invertibility of operators, as has already been mentioned, is to exploit these properties for stability multipliers.

However, it is easy to observe that a straightforward extension of the lemma assures a compatible factorization for every $M \in \mathbb{B}$, whose spectrum does not contain the negative real line including the origin.
${ }^{1}$ Note that even such simple driving-point impedance functions as $\left(s^{2}+a s\right.$ $+b) /\left(s^{2}+c s+d\right)$, for some particular values of the parameters, phase characteristics that touch the boundaries of the strip $(-\pi / 2,+\pi / 2]$ at certain values of the frequeacy. 


\section{REFERENCFS}

[1] G. Zames and P. L. Falb, "Stability conditions for systems with monotone and slope-restricted bonlinearities," SI A.M J. Contr.. vol. 6, pp. 89-108. 1968.

[2] J. C. Willems and R. W. Brockett, "Some new rearrangement inequalities having application in stability analysis" IEEE Trans. Automat Contr. vol. AC-13. pp. 539-519, Oct. 1968

[3] $M . ~ h$. Sandareshan and M. $A$. L. Thathachar, " $L$-stability of linear timerarying systems-Conditions involving noncausal multipliers." $I E E^{\prime} E$ Trar.s. Automat. Contr. vol, AC-17, pp. 504-510, Aug. 1972.

[1] N. l)unford and J. T. Schwartz, Linear Operatore, part 1. New York: In

[5] J. C. Willems, The Analysis of Feedback Systems. Cambridge. Mass.: M.I.T. Press, 1970.

[6] R. W. Brockett and J, L. Willems, "Frequency domain stability criteriaPart I,"IEE' Trans. Automat. Contr., vol. AC-10, pp. 255-261. July 1965 - "Frequency domain stability criteria-Part II." IEEE Trans. Automat. Contr. rol. AC-10, pp. 407-413. Oet. 1965.

[i] MI. A. L. Thathachar, "Stability of systems with power-law nonlinearities," A utomatica. vol. 6, pp. $721-730,1970$.

[8] R. Saeks, "Causality in Hilbert space." SIA.H Rer., vol. 12. pp. 357-383. 1970 .

\section{The Circle Criterion and Quadratic Lyapunov Functions for Stability Analysis}

\section{JACQUES L. WILLEMS}

Abstract-In this note the general equivalence is pointed out between the stability results obtained by means of the circle criterion and those derived from an optimum quadratic Lyapunov function.

In a recent note, the stability results obtained for a second-order system by means of the circle criterion are compared with the stability results derived from an optimum quadratic Lyapunov function [1]. The conclusion that both methods yield equivalent results is valid in general for nonlinear time-dependent feedback systems; this is discussed in this note. Consider the system equations

$$
\dot{x}(t)=A x(t)-b k(x, t) c x
$$

where $A, b$, and $c$ are $(n \times n),(n \times 1)$, and $(1 \times n)$ matrices, respectively, and realize the transfer function $G(s)=c(I s-A)^{-1} b$ in a minimal way [2]. The circle criterion [3] proves asymptotic stability in the large of the null solution of (1) for all $k(x, t)$ such that for an arbitrarily small positive $\epsilon$

$$
a+\epsilon \leqslant k(x, t) \leqslant b-\epsilon
$$

if the function

$$
F(s)=\frac{1+a G(s)}{1+b G(s)}
$$

is positive real. This is also the best stability result that can be abtained from a quadratic Lyapunov function; indeed the following theorem is true.

Theorem 1: There exists a positive-definite quadratic Lyapunov function $V(x)=x^{\prime} P x$, with $P=P^{\prime}>0$, such that the derivative along the solutions of (1)

$$
W(x, t)=x^{\prime}\left[\left(A^{\prime} P+P A\right)-k(x, t)\left(P b c+c^{\prime} b^{\prime} P\right)\right] x
$$

is negative definite for all $k(x, t)$ satisfying (2) if and only if $F(s)$, as defined in (3), is positive real.

This theorem can be proved by means of the Kalman-Yacubovitch-Popov lemma [4], [j] and the necessity of the condition, which is of interest here, is actually contained in Popov's result that the Popov stability criterion for nonlinear feedback sy-stems cannot be improved upon by means of a Lur'e-type Lyapunov function [3]. A proof of the necessity part of the theorem is given by means of the following lemma [6].

Lemma 1: There exists a positive-definite $\boldsymbol{P}$ satisfying the matrix inequality

Manuscript received September 25. 1972. gium.

The author is with the Engineering School, University of Gent, Ghent, Bel-

$$
A^{\prime} P+P A+\left(P b-g c^{\prime}\right)\left(b^{\prime} P-g c\right)-a b c^{\prime} c \leqslant 0
$$

with $g=(a+b) / 2$ only if, for all real $\omega$,

$$
1+(a+b) \operatorname{Re} G(j \omega)+a b|G(j \omega)|^{2} \geqslant 0 .
$$

Proof of Theorem 1: Suppose there exists a positive-definite matrix $P$ satisfying the conditions of the theorem. Then

$$
M(k)=A^{\prime} \dot{P}+P A-k\left(P b c+c^{\prime} b^{\prime} P\right)<0
$$

for $a<k<b$, and at least negative semidefinite for $k$ equal to $a$ or $b$. Since $M(+\infty)$ and $M(-\infty)$ cannot both be negative definite, there exists either a constant $a_{1} \leqslant a$ or a constant $b_{1} \geqslant b$ (or both) such

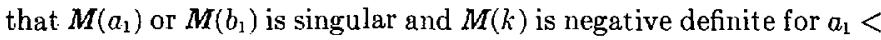
$k<b$ or $a<k<b_{1}$. Suppose $a_{1}$ exists. Then

$$
x^{\prime} M\left(a_{1}\right) x=-\sum_{i=1}^{n-1}\left(z_{i} x\right)^{2}
$$

such that the set $z_{1}, \cdots, z_{n-1}, c$ spans $R^{n}$. Hence, with $r$ positive,

$$
b^{\prime} P=r c+\Sigma m_{i} z_{i} .
$$

It can be assumed without loss of generality that $r=\left(-a_{1}+b\right) / 2$, since this only involves multiplication of $P$ by a positive constant. The negative definiteness of $M(b)$ implies

$$
\left(b-a_{1}\right)\left(\Sigma m_{i} z_{i} x\right)^{2}-2 r \Sigma\left(z_{i} x\right)^{2} \leqslant 0
$$

for all $x$, and hence

$$
A^{\prime} P+P A+\left(P b-\frac{a+b}{2} c\right)\left(b^{\prime} P-\frac{a+b}{2} c^{\prime}\right)-a b c^{\prime} c \leqslant 0
$$

such that Lemma 1 proves that (5) is true. Since the existence of the Lyapunov function implies that

$$
\dot{x}(t)=(\dot{A}-k b c) x(t)
$$

is asymptotically stable for $a<k<b$, and at least weakly stable at $k=a$ and $k=b$, it is concluded that $F(s)$ is positive real.

The proof of sufficiency of the condition can be found in [3] or obtained by reversing the above argument. This shows that the results obtained from the circle criterion for the asymptotic stability in the large of (1) can be matched, but not improved upon, by the use of a quadratic Lyapunov function.

\section{REFERFNCES}

[1] A. C. Tsoi and H. M. Power, "Equiralent predictions of the circle criterion and an optimum quadratic form for a second-order system." $Y E E E$ " Trans. Automat. Contr. (Tech. Notes and Corresp.), vol. AC-17. pp. 565-566, Aug. 1972 .

[2] R. IV. Brockett, Finite Dimensional Linear System., New York: Wiley, 1970 .

[3] J. L. Willems, Stability Theory of Dynamical System: New York: Wiley, 1970 .

[4] R. E. Kalman, "Liapunov functions for the problem of Lur'e in automatic control," Proc. Nat. Acad. Sci. L'.S., vol. 49. pp. 201-205, 1963.

[5] V. M. Popov, "Absolute stability of nonlinear systems oi automatic control." Automat, Remote Contr., vol. 22, pp. 85i-8i5, 1962.

[6] J. C. Willems, "Least squares stationary optimal control and the algebraie Riccati equation," IEEE Trans. A utomat. Contr., vol. AC-16. pp. 621-634, Dec. 1971 .

\section{Forced Oscillations of the van der Pol Oscillator for Large Detuning}

\section{T. W. TANG ANI R. V. MIONOPOLI}

Abstract-Frequency-response curves for the driven van der Pol oscillator are presented which are valid for large detuning. Three different coefficients for the sinusoidal forcing term are considered. It is shown that the theoretical results obtained may be used to accurately predict experimental results. 